\title{
A New Degree of Freedom for Variable Axial Piston Pumps with Valve Plate Rotation
}

\author{
Thomas Heeger and Liselott Ericson \\ Dep. of Management and Engineering, Fluid and Mechatronic Systems, \\ Linköping University, 58183 Linköping, Sweden \\ E-mail: thomas.heeger@liu.se; liselott.ericsson@liu.se
}

\begin{abstract}
Conventionally, variable axial piston machines vary displacement by adjusting the length of the piston stroke, which can be done by adjusting the angle of the swash plate in swash plate machines or the angle of the cylinder block in bent axis machines. Another possibility to achieve variable displacement is to rotate the valve plate and thus adjust the effective use of the piston stroke. An advantage of this method is that it only requires small forces and is easier to control in comparison to conventional displacement adjustment.

This idea is not new, and the concept was studied decades ago, but unfortunately, cavitation and high pressure peaks in the bridge between the kidneys hindered a successful implementation of valve plate rotation.

Using a double pump with opposing pistons offers the potential to overcome these obstacles, as the displacement can be adjusted by joint rotation of both valve plates, and the effective bridge angles can be adjusted by relative rotation of the valve plates. This paper presents a methodology to optimise valve plate kidney angles for a double pump with rotating valve plates. Optimisation results for exemplary sets of operating points are presented. At high setting ratios, power losses and flow pulsations can be reduced. The risk of cavitation and high pressure peaks can be eliminated, but at the expense of cross-porting and increased losses at low setting ratios and high speeds.
\end{abstract}

Keywords: axial piston pump, variable displacement, valve plate rotation, bridge angles, opposing pistons

\section{Introduction}

Conventionally, variable axial piston machines vary displacement by adjusting the length of the piston stroke, which can be done by adjusting the angle of the swash plate in swash plate machines or the angle of the cylinder block in bent axis machines [1]. Another way to achieve variable displacement is to adjust the effective use of the piston stroke, which can be done by rotating the valve plate [2]. It is beneficial that valve plate rotation requires smaller control forces and provides easier controllability in comparison to conventional displacement adjustment [3].

This idea is not new, and the concept was already studied decades ago, but unfortunately, cavitation and high pressure peaks in the bridge between the kidneys have proven to be a major drawback of valve plate rotation [4]. Further issues that need to be considered are noise and backflow [5].

When using a double pump with opposing pistons, two rotating groups, and therefore also two valve plates, are used, see fig. 1a. When both rotating groups share a common cylinder barrel, and opposing pistons are connected through the cylinder barrel, each of the valve plates affects the effective port angles. Therefore, the ability to rotate each valve plate independently gives an additional degree of freedom, which can be used to adjust the preand decompression angle for different operating points and for different setting ratios, see fig. $1 \mathrm{~b}$. This idea has recently been presented and patented in [6].

This paper investigates whether this degree of freedom can be used to overcome the issues stated above on the example of an axial piston pump of the swash plate type. Basic considerations for port design and the state of the art for valve plate rotation (for pumps with one valve plate) are summarised in sec. 2. A double pump with 
opposing pistons and rotating valve plates is simulated, and a methodology to optimise valve plate kidney angles is suggested in sec 3. In section 4, optimisation results are presented. The results are discussed in sec. 5, and the most important findings are summarised in sec. 6.

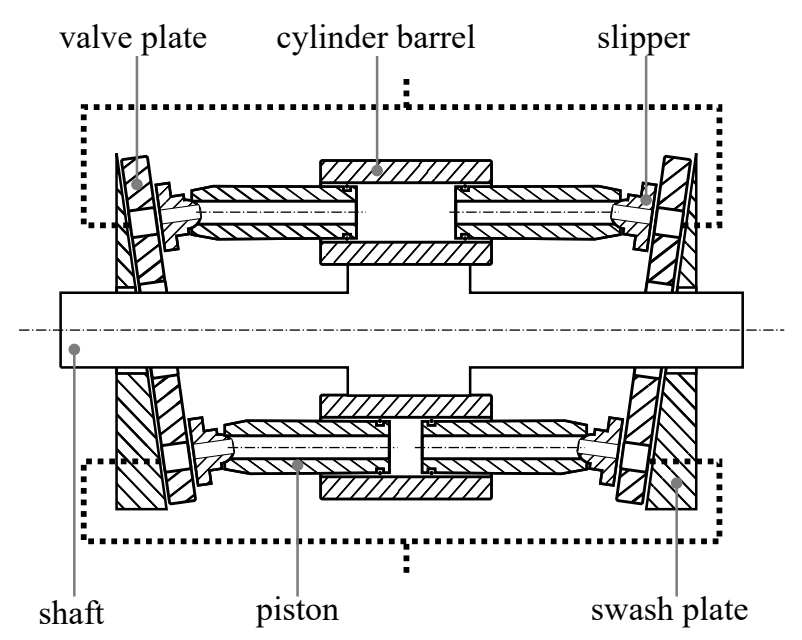

(a) Axial piston machine with opposing pistons. Two opposing pistons share one bore in the cylinder barrel, and therefore they communicate with one another. The flows through the corresponding kidneys in the valve plates are combined, so the machine possesses only one inlet channel and one outlet channel.

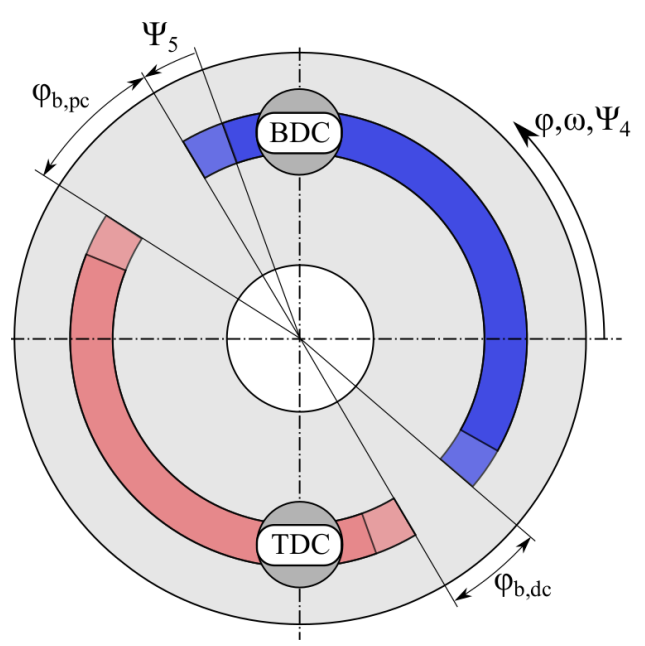

(b) Effective displacement is adjusted by the joint valve plate rotation angle $\Psi_{4}$. The relative valve plate rotation angle $\Psi_{5}$ gives an additional degree of freedom. This can be used to choose the effective bridge angles for pre-compression $\varphi_{b, p c}$ and for de-compression $\varphi_{d, p c}$ depending on the operating condition. The low pressure kidneys are shown in blue/dark grey and the high pressure kidneys are shown in red/light grey. $\varphi$ and $\omega$ are the shaft rotation angle and angular speed.

Figure 1: Sketch of double pump with opposing pistons and the additional degree of freedom due to having two valve plates.

\section{State of the Art}

In the following, the basics of port design (sec. 2.1) and valve plate rotation for machines with one valve plate (sec. 2.2) are summarised, so that the background for the idea of adding a degree of freedom by using a double pump with opposing pistons in sec. 3 is given.

\subsection{Basics of Port Design}

In positive displacement pumps, the displacement volume needs to be connected to the low pressure (LP) kidney to take in fluid and the high pressure (HP) kidney to deliver the fluid. So-called bridges separate the two kidneys and prevent cross-porting (i.e., flow from the HP kidney to the LP kidney through a direct connection provided by the displacement volume) at commutation. Typically, commutation takes place around the dead centres, where the axial piston speed is low [4]. In axial piston machines, the kidneys are located in the valve plates, which are designed to separate the inlet channel from the outlet channel and to provide a smooth pressure transition for each piston chamber. A fixed valve plate cannot provide a smooth pressure transition for all operating conditions [7]. Figure 2 shows a zero-lapped valve plate and the corresponding flow into and out of one pump chamber. In a zero-lapped valve plate, the bridge between the HP kidney and the LP kidney covers exactly the opening of one displacement volume. That means that the zero-lapped valve plate separates the HP kidney from the LP kidney, but no pre- or decompression takes place. This leads to flow pulsations due to the compressibility of the fluid.

To calculate the flow for one displacement volume (i.e., in case of axial piston pumps for each cylinder), the continuity equation can be used [8]:

$$
\sum q=\frac{\mathrm{d} V_{\text {cyl }}}{\mathrm{d} t}+\frac{V_{\text {cyl }}}{\beta_{\mathrm{e}}} \frac{\mathrm{d} p}{\mathrm{~d} t}
$$

The first summand of eq. 1 represents the kinematic flow, whereas the second summand represents the compressible flow, with $\beta_{\mathrm{e}}$ being the effective bulk modulus, $\frac{\mathrm{d} p}{\mathrm{~d} t}$ being the rate of pressure change inside the cylinder, and $V_{\text {cyl }}$ being the cylinder volume. For axial piston machines of the swash plate type, the cylinder volume can be calculated as

$$
V_{\text {cyl }}=V_{\text {dead }}+(1+\sin \varphi) \tan \alpha R_{\mathrm{b}} A_{\mathrm{p}}
$$




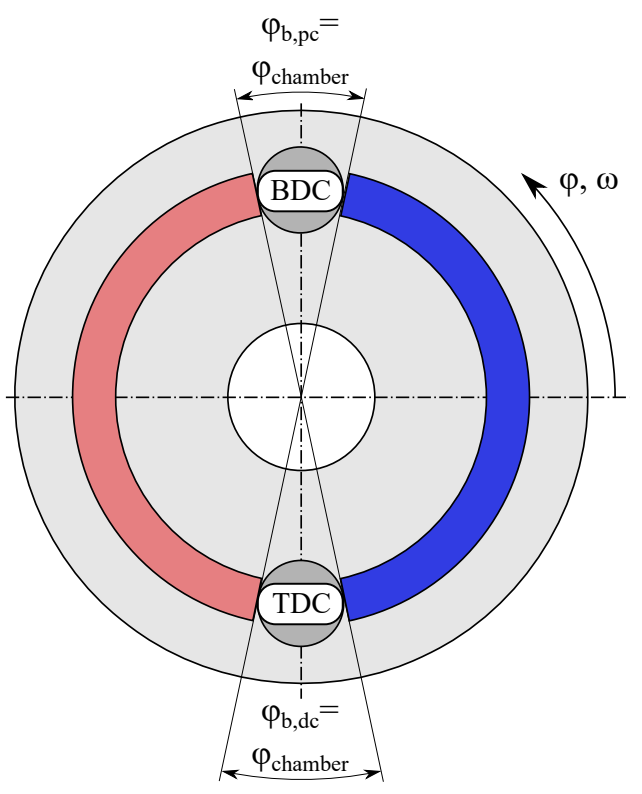

(a) Zero-lapped valve plate.

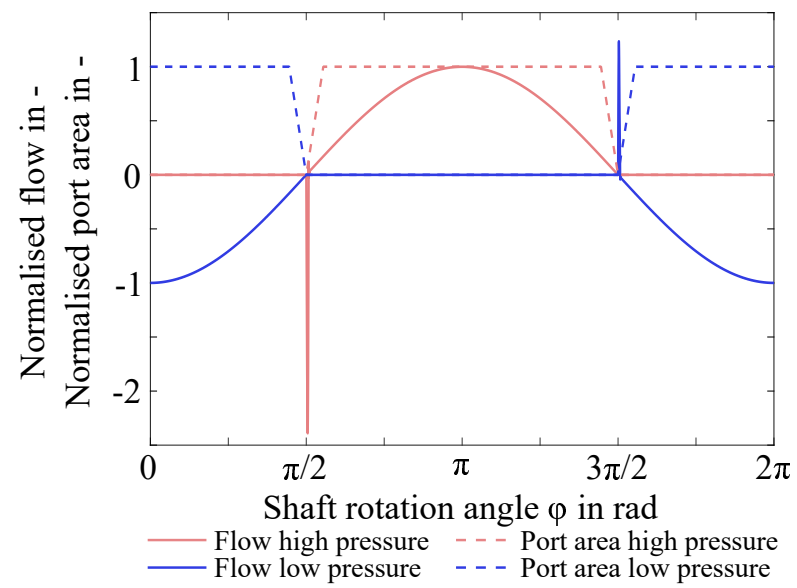

(b) Exemplary flow for one pump chamber for a pump with a zero-lapped valve plate. Positive flow is the flow that is delivered by a pump (i.e., the flow that goes out of the pump chamber). At commutation, sharp flow peaks occur due to the compressibility of the fluid in the pump chamber.

Figure 2: For a zero-lapped valve plate, the bridge angles $\varphi_{b, p c}$ and $\varphi_{b, d c}$ are identical with the swept angle over one of the openings from a displacement chamber $\varphi_{\text {chamber }}$ to the valve plate. In this figure, commutation takes place at bottom dead center and top dead center. At commutation, pressure equalisation leads to compressible flow pulsations.

with the cylinder's dead volume $V_{\mathrm{dead}}$, the shaft rotation angle $\varphi$, the swash plate angle $\alpha$, the barrel radius $R_{\mathrm{b}}$ and the piston area $A_{\mathrm{p}}$. Thus, the kinematic flow is

$$
\frac{\mathrm{d} V_{\mathrm{cyl}}}{\mathrm{d} t}=\omega \cos \varphi \tan \alpha R_{\mathrm{b}} A_{\mathrm{p}}
$$

In order to reduce compressible flow pulsations in fluid power machines, pre-compression takes place before the displacement volume connects to the HP kidney and de-compression takes place before the displacement volume connects to the LP kidney. The angle at bottom dead center (BDC) is larger than the angle at top dead center (TDC), as the fluid volume at BDC is larger [2]. The principle design for a valve plate depending on the operating quadrant can be seen in fig. 3 .

The quasi-static pressure change during pre- and de-compression with eq. 1 and $\sum q=0$ can be calculated as

$$
p_{2}-p_{1}=\beta_{\mathrm{e}} \ln \frac{V_{\mathrm{cyl}, 1}}{V_{\mathrm{cyl}, 2}}
$$

with the index 1 representing the state when entering the bridge and index 2 representing the state when leaving the bridge.

Equation 4 shows that pre- and decompression as shown in fig. 3 can only be customised to one pressure level. For this reason, pressure relief grooves are typically used. They provide a small channel, which smoothens the pressure transition for a wider range of pressure levels. However, the flows through the pressure relief grooves lead to undesired losses, so that pressure relief grooves are seen as a "necessary evil" [7].

\subsection{Displacement Control Through Valve Plate Rotation for Pumps with One Valve Plate}

In [2], the concept of displacement control through valve plate rotation has been summarised. Figures 4 and 5 visualise the principle: When the commutation is moved away from TDC and BDC, the kinematic flow while connected to the HP kidney becomes negative for some degrees of shaft rotation, and thus the effective stroke is reduced. This enables valve plate rotation to continuously adjust the effective displacement, and it is possible to switch between pump and motor mode. The setting ratio $\varepsilon$ of the machine follows eq. 5

$$
\varepsilon=\cos \varphi_{\mathrm{VP}}
$$

where $\varphi_{\mathrm{VP}}$ is the valve plate rotation angle. 


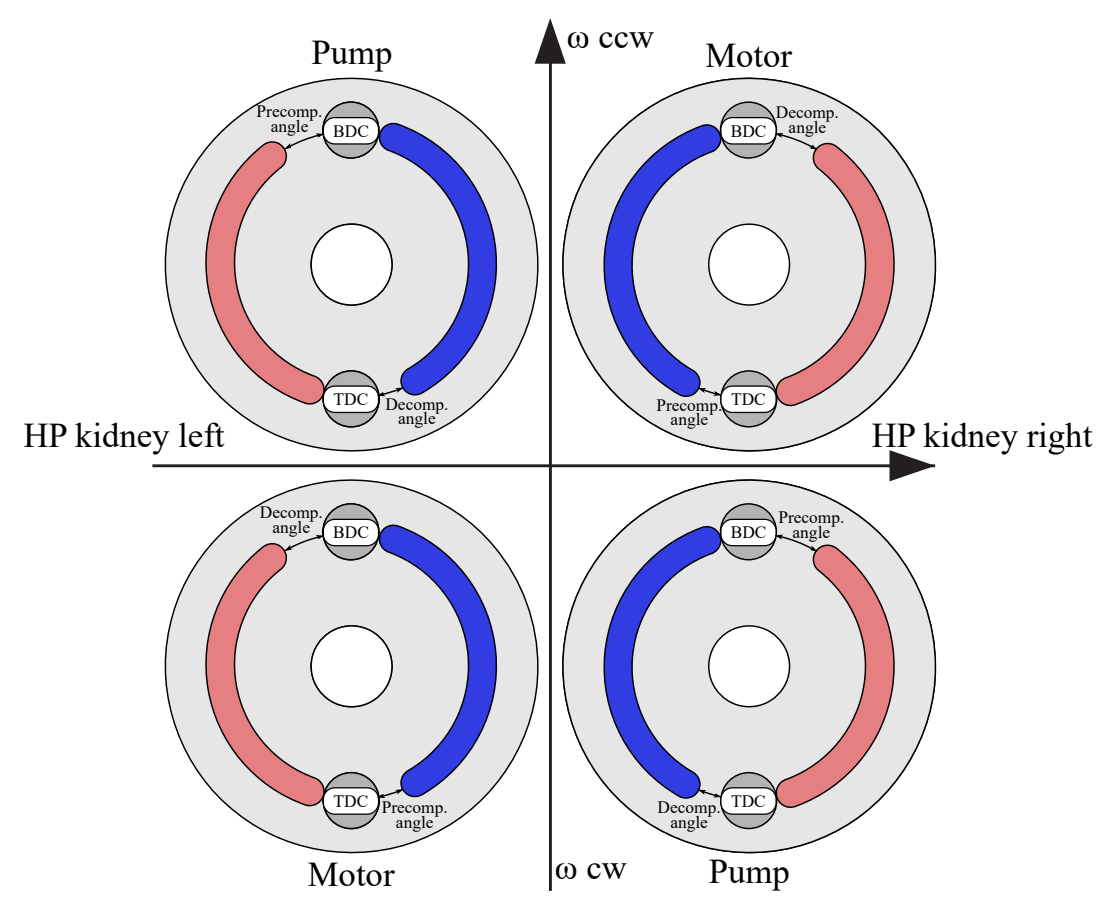

Figure 3: Principle design of optimised valve plate design for different operating quadrants for a fluid power pump/motor [2]. The bridge angles are larger than $\varphi_{\text {chamber, }}$, therefore pre- and decompression take place. Compressible flow pulsations are reduced by pre-and decompression during commutation.

A challenge for displacement control via valve plate rotation is caused by the commutation and pre- and decompression angles, as the commutation does not take place around the dead centres. Differentiating eq. 2 with respect to the shaft rotation angle $\varphi$ delivers the change of a chamber volume by $\varphi$ :

$$
\frac{\mathrm{d} V_{\mathrm{cyl}}}{\mathrm{d} \varphi}=\cos \varphi \tan \alpha R_{\mathrm{b}} A_{\mathrm{p}}
$$

Equation 6 shows that $\left|\frac{\mathrm{d} V_{\text {cyl }}}{\mathrm{d} \varphi}\right|$ is largest at $\varphi=0$ and $\varphi=\pi$, whereas it is zero at $\varphi=\pi / 2$ and $\varphi=3 / 2 \pi$ (where commutation usually takes place). For this reason, the same pre-/de-compression angles lead to higher maximum and lower minimum pressures, when the commutation takes place at greater distance from the dead centres (see eq. 4).

Additionally, the kidney opening/closing takes place gradually. Assuming a positive overlap, the kidney opening reduces to $0 \mathrm{~m}^{2}$ when a cylinder enters a bridge. Especially during the end of the closing process, a significant throttling effect appears, which is reinforced by high axial piston velocities [4]. Due to this throttling effect,

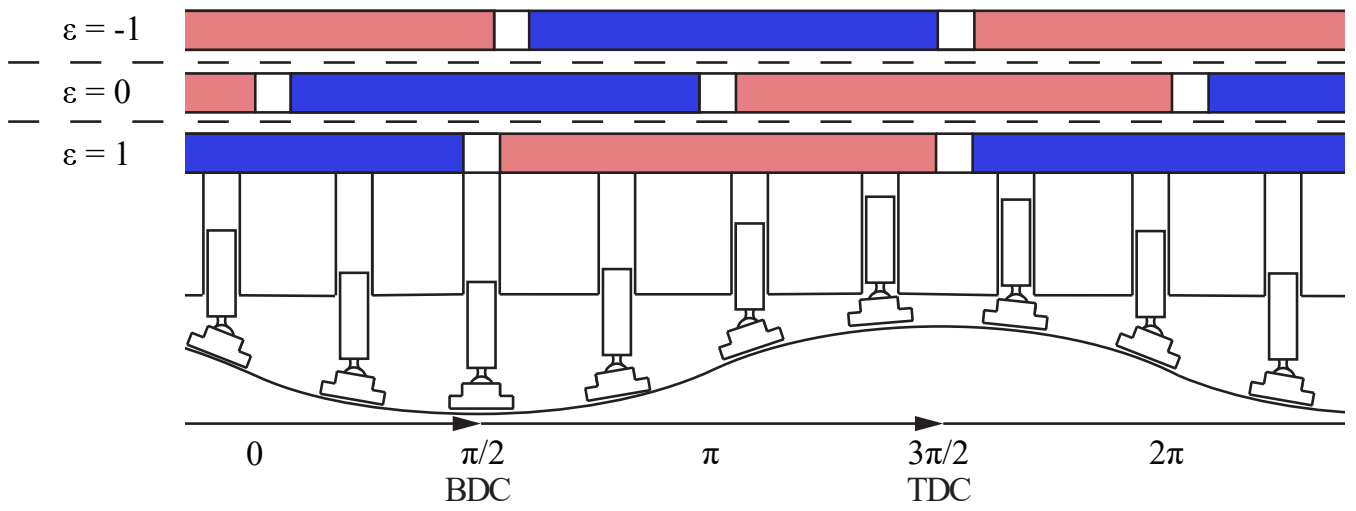

Figure 4: A valve plate in full motor-mode $(\varepsilon=-1)$, no-flow-mode $(\varepsilon=0)$ and full pump-mode $(\varepsilon=1)$. Red/light grey rectangles symbolise the high-pressure kidney and blue/dark grey rectangles symbolise the low-pressure kidney for one full shaft rotation from 0 to $2 \pi[2]$. 


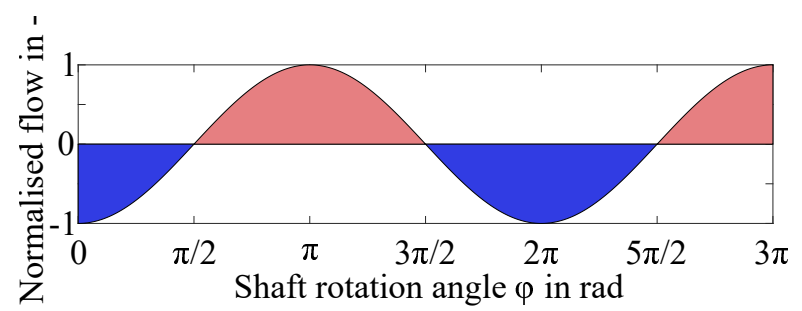

(a) Flow at zero valve plate rotation, hence full displacement.

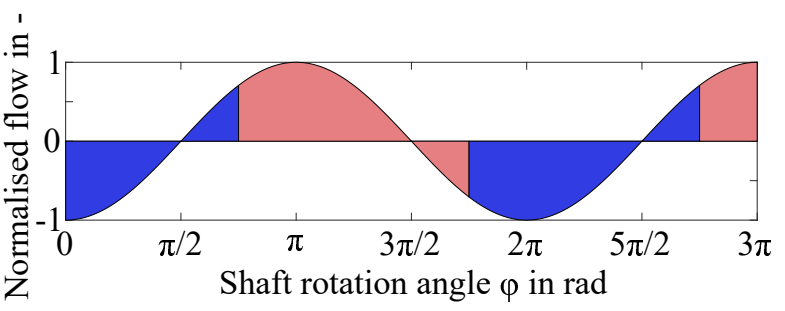

(b) Flow with $45^{\circ}$ valve plate rotation, hence $\varepsilon=0.7$.

Figure 5: Normalised kinematic flow from one cylinder. Blue/dark grey areas are the volumes entering the cylinder from the low-pressure kidney and red/light grey are the volumes leaving the cylinder to the high-pressure kidney [2].

[7] states that it is "never a good idea" to even place one of the support ribs (which are typically used to improve the structural stability of the valve plate on the HP side) at the location of maximal axial piston speed.

The severity of this phenomenon is visualised in fig. 6 , which shows the maximal and minimal chamber pressures occurring for zero-lapped valve plates during one shaft revolution, over the valve plate position. The maximal and minimal pressures occur at the reduced port area around commutation, due to the above described throttling effect. For a valve plate rotation of 0 or $\pi$, commutation takes place at the dead centres, hence at minimal axial piston speed. For a valve plate rotation of $\pi / 2$ or $3 \pi / 2$, commutation is moved to a maximum distance from the dead centres, and so also to the location of maximum axial piston speeds and the maximum values of $\left|\frac{\mathrm{d} V_{\mathrm{cyl}}}{\mathrm{d} \varphi}\right|$. Therefore, the risk of cavitation and high pressure peaks is increased. Of course, higher pump speeds also lead to higher piston speeds, as well as increasing maximal pressures and decreasing minimal pressures.

Between $\pi$ and $2 \pi$ valve plate rotation, the maximal pressure is higher than between 0 and $\pi$, because the chamber is loaded with HP before the commutation between BDC and TDC (where the port restriction leads to pressure overshoots). Analogous considerations can be made for pressure undershoots in the LP kidney. When the chamber volume is increased (between TDC and BDC) around commutation, the port restrictions lead to pressure undershoots. These undershoots are more profound when the chamber is already at LP before commutation. When evaluating the pressure undershoots, it must be considered that a simplified calculation of the bulk modulus is applied. More details on the bulk modulus calculation can be found in sec. 3.1.
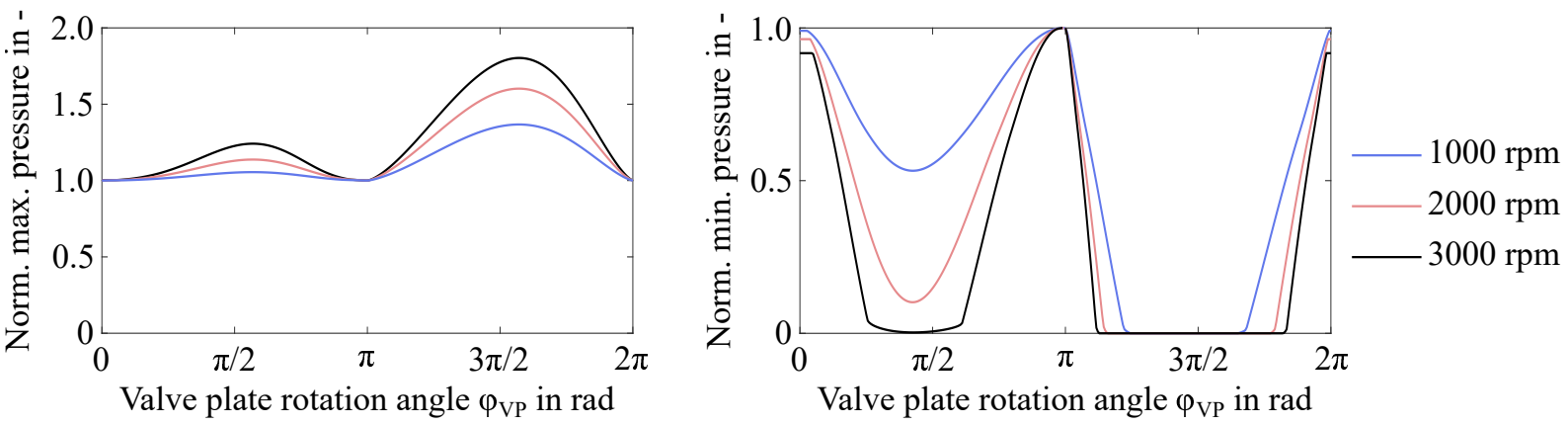

Figure 6: Throttling effect for zero-lapped valve plate: maximal and minimal pressures in chambers during one shaft revolution over angle of valve plate rotation. These graphs correspond to the machine described in sec. 3.1, with both valve plates being zero-lapped and in identical positions.

\section{Valve Plate Rotation for a Double Pump with Opposing Pistons}

In order to evaluate the potential of a double pump of opposing piston type with two valve plates, a simulation model is created. Optimisation is used to find the optimal kidney angles and valve plate rotation angles for given sets of operating points. Simulation results are evaluated by an objective function.

\subsection{Simulation Model}

A Hopsan model is used to simulate the steady-state behaviour of the pump for given kidney and valve plate rotation angles. Hopsan is a one-dimensional multi-domain simulation tool using the transmission line theory. Figure 7 illustrates the pump simulation model used in this paper. For each displacement chamber, the model consists of a volume, which is connected to the HP source resp. the LP source through orifices. As this paper investigates a double pump with opposing pistons, see fig. 1a, two pistons share one displacement volume. Each 
displacement volume is connected to both valve plates, and each valve plate has two kidneys. The ends of the kidneys are rectangular, in order to provide a larger passage area than circular ends. The size of each displacement volume depends on the shaft angle, and the area and circumference of each orifice depend on the shaft rotation angle and the valve plate rotation angles.

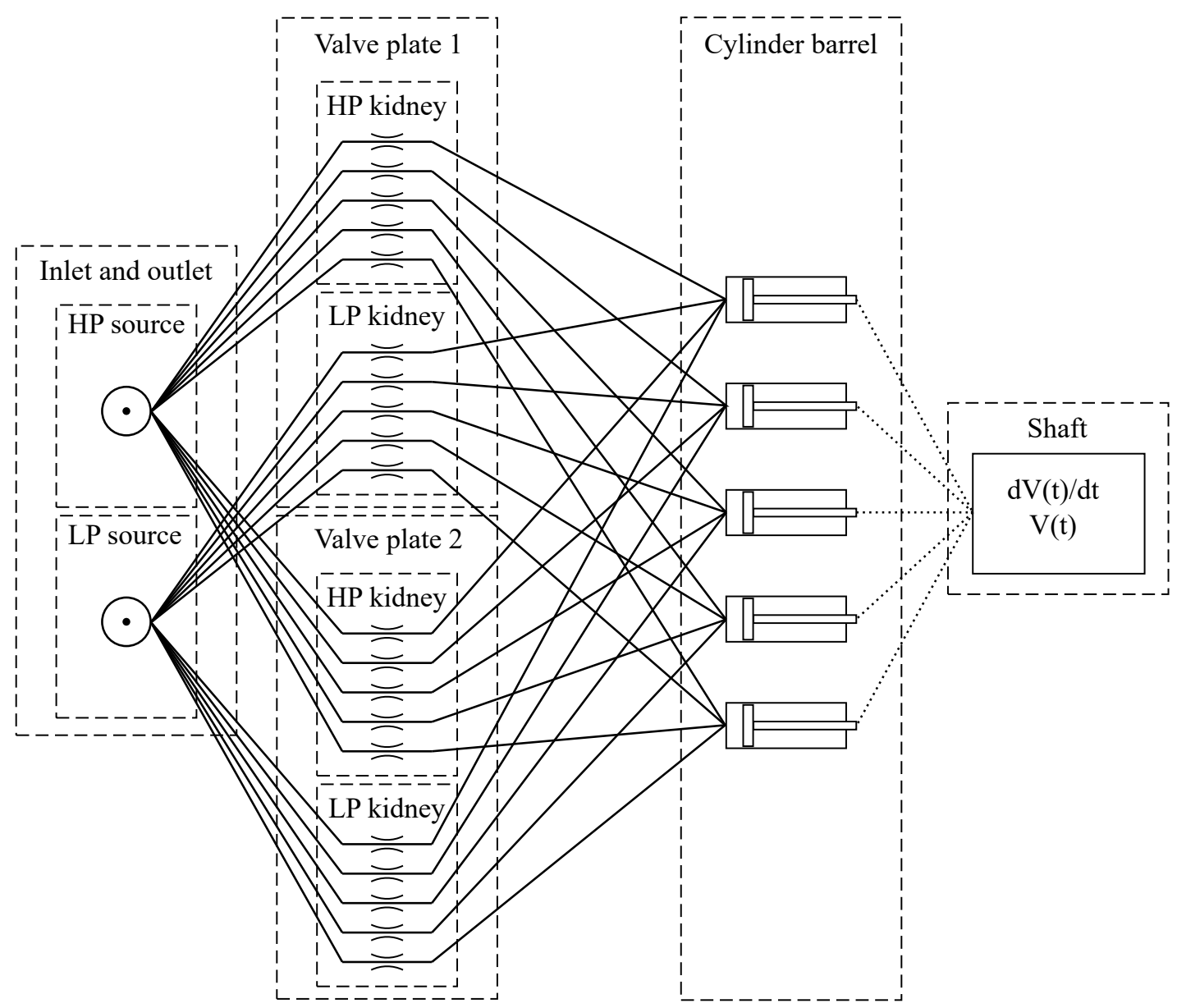

Figure 7: Illustration of pump model structure. Of course, any number of cylinders can be implemented.

The fluid's effective bulk modulus $\beta_{\mathrm{e}}$ for each displacement chamber is calculated as

$$
\beta_{\mathrm{e}}=\frac{\beta_{\mathrm{oil}}}{1+\frac{x_{0}}{\kappa p} \frac{\beta_{\mathrm{oil}}}{1-x_{0}}\left(\frac{p_{0}}{p}\right)^{\frac{1}{\kappa}}}
$$

with $\beta_{\text {oil }}$ being the oil's bulk modulus, $x_{0}$ being the volume fraction of undissolved air at reference pressure $p_{0}$, $p$ being the pressure in the chamber and $\kappa$ being the isentropic expansion factor of air.

Eq. 7 assumes that both the oil and the undissolved air change their volume based on the respective bulk moduli, but no air is dissolved or released. This neglects the phenomenon of cavitation, which describes the release of air from oil below ambient pressure, which reduces the bulk modulus [9]. As cavitation is neglected in this paper, the pressure undershoots are overestimated (i.e., considering cavitation would lead to higher minimum pressure levels).

The most relevant parameters of the simulated machine are shown in tab. 1.

\subsection{Optimisation}

In this paper, the kidney angles are defined by the relative position of the kidneys and their opening and closing to one another. In combination with the joint valve plate rotation angle and the relative valve plate rotation angle, the effective port angles are defined.

To design the valve plates, there are three degrees of freedom for the kidney angles of the valve plate, as there are three relative positions to be chosen for the kidneys' opening and closing angles. In this paper, both valve plates have the same kidney angles in order to simplify the problem. Furthermore, pressure relief grooves are not 
Table 1: Parameters of simulated machine.

\begin{tabular}{cc}
\hline Quantity & Value \\
\hline Type & Swash plate \\
Displacement volume & $35 \mathrm{~cm}^{3} / \mathrm{rev}$ \\
Number of pistons & $2 \times 11$ \\
Swash plate angle & $8 \mathrm{deg}$ \\
Dead volume ratio & 0.70 \\
Swept chamber angle & $26.9 \mathrm{deg}$ \\
\hline
\end{tabular}

investigated. Additional to the three degrees of freedom for the kidney angles there are two degrees of freedom for the valve plate rotation angles (one for their joint rotation, and one for their relative rotation).

In order to solve this multi-dimensional optimisation problem, the complex-RF optimisation algorithm is used. Complex-RF is a single objective, non-gradient optimisation algorithm. It modifies Box's complex method [10] by adding a randomisation factor to avoid pre-mature collapsing of the complex and a forgetting factor which ensures that older parameter sets are replaced by more recent ones [11].

The operating points considered in this paper are shown in tab. 2. In this paper, speed and pressure are fixed, but the outlet flow is varied between different setting ratios. An explicit weighting or trade-off between the operating points does not take place in this paper, i.e., each operating point has the same weight.

Table 2: Operating points considered for valve plate optimisation. A setting ratio of 100\% of the ideal flow cannot be reached due to the compressibility of the fluid. Therefore, as reference for "full" displacement, the outlet flow of non-rotated zero-lapped valve plates is used.

\begin{tabular}{cc}
\hline Quantity & Value \\
\hline Setting ratio & $0.9862,0.75,0.5,0.25,0$ \\
Speed & separate optimisations for: $1500 \mathrm{rpm}, 3000 \mathrm{rpm}$ \\
Outlet pressure & $15 \mathrm{MPa}$ \\
Inlet pressure & $0.5 \mathrm{MPa}$ \\
Eolume ratio of undissolved air at $0.1 \mathrm{MPa}$ & 0.01 \\
Effective bulk modulus & $1.8 \mathrm{GPa}$ \\
\hline
\end{tabular}

\subsubsection{Setup of Optimisation}

The preprocessing, optimisation algorithm and post-processing are implemented in MATLAB. The simulation is carried out in Hopsan through the Hopsan API. The variables $\Psi_{1}-\Psi_{5}$ are sketched in fig. 8 and summarised in tab. 3.

The optimisation procedure is illustrated in fig. 9. The angle $\varphi_{\text {ref }}$ is chosen arbitrarily during pre-processing. The variables $\Psi_{1}-\Psi_{3}$ define the kidney angles and they are the optimisation variables for the outer optimisation loop. As the pressures at commutation are highly dependent on the bridge angles, $\Psi_{1}$ and $\Psi_{3}$ are chosen to explicitly represent bridge angles, rather than choosing an absolute reference for each kidney start/end angle and thus implicitly defining the bridge angles.

For a given set of kidney angles, the ideal valve plate rotational angles $\Psi_{4}$ and $\Psi_{5}$ for each operating point must be found in an inner optimisation loop. $\Psi_{4}$ represents the joint rotation of both valve plates, which mainly affects the effective displacement of the machine, whereas $\Psi_{5}$ represents the relative rotation between the two valve plates, which mainly effects the effective pre- and de-compression angles.

The objective function values of the inner loop are returned to the outer loop. The objective function value of the outer loop is the sum of the objective function values of the inner optimisation loop (one value for each operating point). Thus the potential of the kidney angles can be evaluated.

\subsubsection{Formulation of Objective Function}

The valve plates (in combination with their rotations) must fulfil several goals, which are combined into one objective function as Complex-RF is a single-objective optimisation method. The aim is to minimise power losses as well as flow pulsations (both in the HP channel and the LP channel). The constraints are as follows: the correct flow level must be provided in the HP channel, the minimum pressure is not to go below a defined threshold and a 

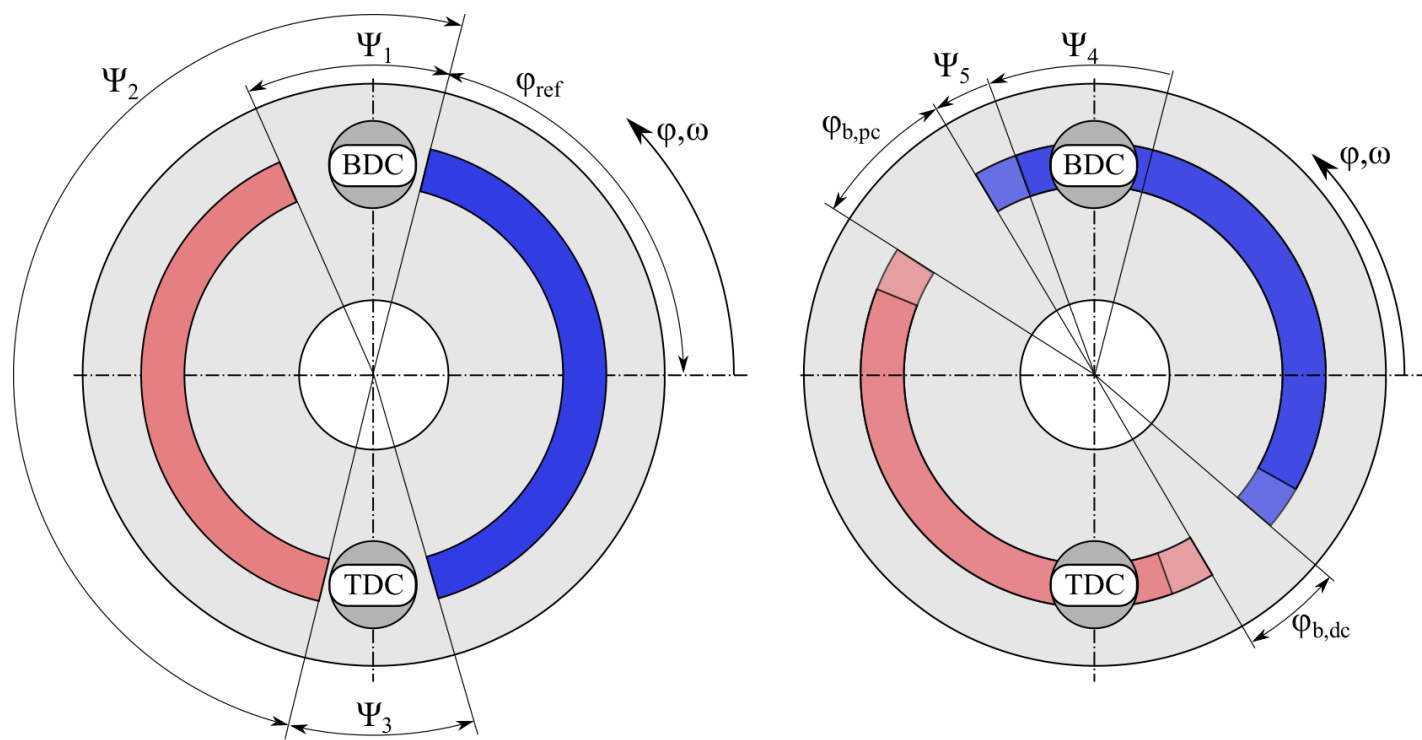

Figure 8: Sketch of optimisation variables. Left: both valve plates are in the same position. Right: Both valve plates are rotated jointly by $\Psi_{4}$, and one valve plate is rotated further by $\Psi_{5} . \varphi_{\text {ref }}$ is set arbitrarily; $\Psi_{1}-\Psi_{3}$ are optimised in the outer loop, and $\Psi_{4}-\Psi_{5}$ are optimised in the inner loop.

Table 3: Optimisation variables

\begin{tabular}{ccc}
\hline Symbol & Quantity & Unit \\
\hline$\Psi_{1}$ & Bridge angle between closing LP kidney and opening HP kidney & deg \\
$\Psi_{2}$ & Angle between closing of LP kidney and closing of HP kidney & deg \\
$\Psi_{3}$ & Bridge angle between closing HP kidney and opening LP kidney & deg \\
$\Psi_{4}$ & Joint rotation angle of both valve plates & deg \\
$\Psi_{5}$ & Relative rotation angle between the valve plates & deg \\
\hline
\end{tabular}

defined maximum pressure is not to be exceeded. The constraints are implemented as soft constraints, i.e., they are implemented as part of the objective function. Equation 8 shows the objective function of the outer loop, which is the sum of the inner loop objective function values of the individual operating points.

$$
f_{\text {total }}\left(\Psi_{1}, \ldots, \Psi_{5}\right)=\sum_{\text {OP }} f_{\text {innerLoop }}
$$

Equation 9 is the objective function of the inner loop, which consists of the constraints as well as the objectives. The constraints are formulated with an exponential increase of objective function value when they are violated, so that the objective function value increases drastically in case of constraint violation.

$$
f_{\text {innerLoop }}\left(\Psi_{1}, \ldots, \Psi_{5}\right)=f_{\mathrm{P}, \text { losses }}+f_{\mathrm{q}, \mathrm{pulse}, \mathrm{HP}}+f_{\mathrm{q}, \mathrm{pulse}, \mathrm{LP}}+f_{\mathrm{p}, \min }+f_{\mathrm{p}, \max }+f_{\mathrm{q}, \text { mean }, \mathrm{HP}}
$$

In the following, the mathematical formulation of the inner loop's objective function and its components are explained in eq. 10 to 16 . Parameters named $f$ represent function values for the respective part of the objective function, parameters named $g$ represent linear weighting factors and parameters named $h$ represent weighting factors in the exponent of an exponential function.

\section{Objectives}

The power losses are calculated from the hydraulic and mechanical power. The function value for power losses increases linearly with the power losses, see eq. 10.

$$
f_{\mathrm{P}, \text { losses }}\left(\Psi_{1}, \ldots, \Psi_{5}\right)=g_{\mathrm{P}, \text { losses }}\left(p_{\text {high }} q_{\text {mean }, \mathrm{HP}}-p_{\text {low }} q_{\text {mean }, \mathrm{LP}}-T \omega\right)
$$

The flow pulsations in the HP channel are calculated as the difference between the highest instantaneous flow and the lowest instantaneous flow in the HP channel. The function value for HP flow pulsations increases linearly with 


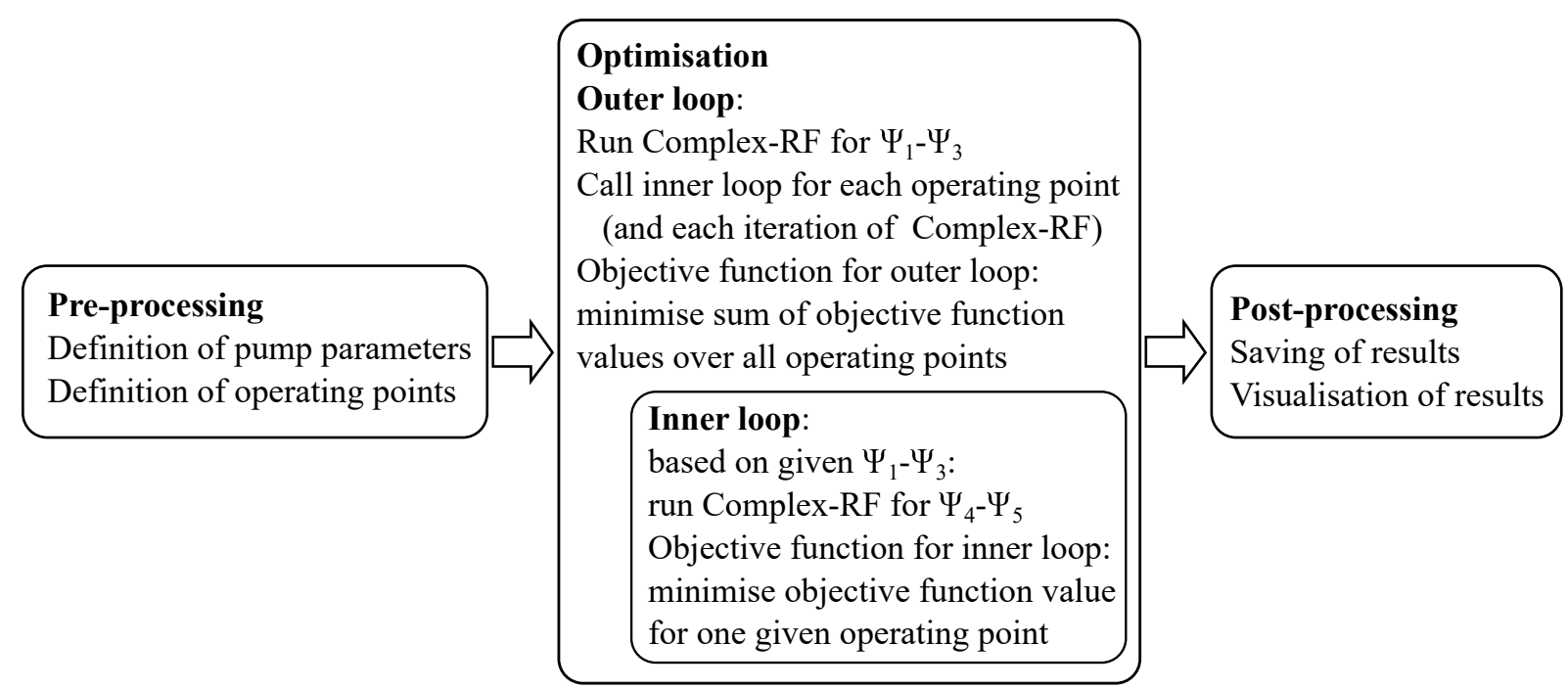

Figure 9: Setup of optimisation.

the peak-to-peak value of the flow, see eq. 11.

$$
f_{\text {q,pulse } \mathrm{HP}}\left(\Psi_{1}, \ldots, \Psi_{5}\right)=g_{\mathrm{q}, \mathrm{pulse}, \mathrm{HP}}\left(\max \left(q_{\mathrm{HP}}\right)-\min \left(q_{\mathrm{HP}}\right)\right)
$$

The flow pulsations in the LP channel and their function value are calculated analogous to the flow pulsations in the HP channel, see eq. 12.

$$
f_{\mathrm{q}, \text { pulse } \mathrm{LP}}\left(\Psi_{1}, \ldots, \Psi_{5}\right)=g_{\mathrm{q}, \mathrm{pulse}, \mathrm{LP}}\left(\max \left(q_{\mathrm{LP}}\right)-\min \left(q_{\mathrm{LP}}\right)\right)
$$

In this paper, the weighting factors $g_{\mathrm{P}, \text { losses }}$ and $g_{\mathrm{q}, \mathrm{pulse}, \mathrm{HP}}$ are chosen so that the function values $f_{\mathrm{P}, \text { losses }}$ and $f_{\mathrm{q}, \mathrm{pulse}, \mathrm{HP}}$ are in the same order for a pump running at full displacement. In comparison to the HP channel flow pulsations, the energy level of LP channel flow pulsations is significantly lower. LP channel flow pulsations are therefore considered less relevant, thus $g_{\text {q,pulse,LP }}<g_{\text {q,pulse,HP. }}$.

A methodological trade-off between the power losses, and the flow pulsations in the HP and LP channel is not considered in this paper.

[12] investigates Pareto fronts for the multi-objective optimisation of fluid power machines and shows that the Pareto fronts for the trade-off between two performance values (e.g., flow pulsations in the HP channel vs. the LP channel) can be quite sharp. That means that a large improvement in one performance value can be enabled without a significant deterioration of the other performance value. By including the above named quantities in the objective function, unnecessarily poor values for individual quantities can be avoided, even when no methodological trade-off is carried out.

\section{Soft constraints}

A defined minimum pressure level must be guaranteed, so that cavitation is avoided. This is necessary because cavitation causes noise and can damage the machine. To penalise cavitation, eq. 13 is implemented: When the minimum pressure is above the threshhold $p_{\text {cav }}$, the function value $f_{\mathrm{p}, \min }$ is 0 . In this paper, $p_{\text {cav }}$ is set to $0.05 \mathrm{MPa}$. When minimum pressure is between $0 \mathrm{MPa}$ and $p_{\mathrm{cav}}, f_{\mathrm{p}, \min }$ increases exponentially with increasing distance from $p_{\text {cav }}$. Due to the simplified bulk modulus calculation (see sec. 3.1), it is possible that absolute pressures of $0 \mathrm{MPa}$ are calculated. When a pressure of $0 \mathrm{MPa}$ is provided as a simulation result, it is important that solutions that are further away from being cavitation-free have increased objective function values, so that the algorithm can find the direction to better solutions. For this purpose, the effective closing angle of the low-pressure kidney $\left(\varphi_{\text {ref }}+\left(\Psi_{4}+\right.\right.$ $\left.\Psi_{5}\right)$ ) and the effective length of the bridge before entering the low-pressure kidney $\left(\Psi_{3}-\Psi_{5}\right)$ are used as proxies, see eq. 14.

$$
f_{\mathrm{p}, \min }\left(\Psi_{1}, \ldots, \Psi_{5}\right)=\left\{\begin{array}{lr}
0, & \text { for } p_{\min } \geq p_{\text {cav }} \\
g_{\mathrm{p}, \text { min }}\left(\mathrm{e}^{h_{\mathrm{p}, \min }\left(p_{\text {cav }}-p_{\min }\right)}-\mathrm{e}^{0}\right), & \text { for } 0<p_{\min } \leq p_{\text {cav }} \\
g_{\mathrm{p}, \text { min }}\left(\mathrm{e}^{h_{\mathrm{p}, \min } p_{\text {cav }}}-\mathrm{e}^{0}+f_{\text {decomp }}+f_{\text {close }, \mathrm{LP}}\right), & \text { for } p_{\min }=0
\end{array}\right.
$$

with 


$$
\begin{aligned}
& f_{\text {decomp }}\left(\Psi_{3}, \ldots, \Psi_{5}\right)=g_{\text {decomp }} e^{h_{\text {decomp }}\left(\Psi_{3}-\Psi_{5}\right)}
\end{aligned}
$$

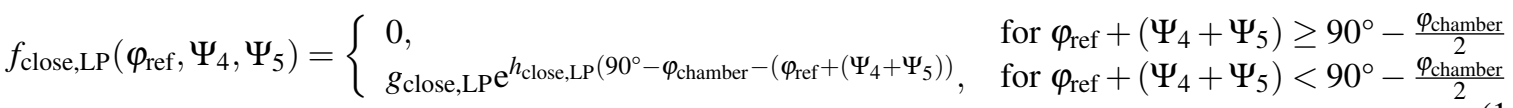

Pressure peaks need to be limited because they can cause noise, vibrations and damages. Pressure peaks that exceed the delivery pressure by a certain percentage are considered as acceptable and therefore receive a function value $f_{\mathrm{p}, \max }$ of 0 , see eq. 15 . In this paper, $k_{\mathrm{HP}}$ is chosen to be 1.2, i.e., a pressure overshoot of $20 \%$ is allowed. Higher pressures lead to an increasing objective function value. As pressure peaks increase aggressively when too much pre-compression takes place, no exponential function is applied here.

$$
f_{\mathrm{p}, \max }\left(\Psi_{1}, \ldots, \Psi_{5}\right)= \begin{cases}0, & \text { for } p_{\max } \leq k_{\mathrm{HP}} p_{\text {high }} \\ g_{\mathrm{p}, \max }\left(p_{\mathrm{max}}-k_{\mathrm{HP}} p_{\text {high }}\right), & \text { for } p_{\max }>k_{\mathrm{HP}} p_{\text {high }}\end{cases}
$$

The desired flow level needs to be provided. Therefore, any deviations from the objective flow level lead to an increasing objective function value $f_{\mathrm{q}, \mathrm{mean}, \mathrm{HP}}$, see eq. 16 .

$$
f_{\mathrm{q}, \text { mean, } \mathrm{HP}}\left(\Psi_{1}, \ldots, \Psi_{5}\right)=g_{\mathrm{q}, \text { mean,HP}}\left(\mathrm{e}^{h_{\mathrm{q}, \text { mean,HP }}\left|q_{\text {mean,HP,expected }}-q_{\text {mean, } \mathrm{HP}}\right|}-\mathrm{e}^{0}\right)
$$

\subsection{Joint Rotation of Zero-lapped Valve Plate as Reference}

In order to evaluate the quality of the results of valve plate rotation, a "benchmark" is needed. For this benchmark, the pump as described in sec. 3.1 is equipped with two zero-lapped valve plates, which are rotated jointly. The valve plate position is chosen so that the flow objectives as described in tab. 2 are met. The small deviations of the valve plate positions in comparison to eq. 5 originate from compressible flow.

\section{Results}

In this section, the optimisation results for both the zero-lapped valve plates and optimised solutions are presented.

\subsection{Joint rotation of zero-lapped Valve Plates}

As stated in sec. 3.3, joint rotation of zero-lapped valve plates is used as a comparison for each operating point. The angular positions of the kidneys are visualised in fig. 10. The corresponding objective function values are shown in fig. 14 and 15. The most important results are summarised in tab. 4 and 6.

At low speeds, the losses at full displacement are high due to insufficient pre-compression resulting in large backflow. The objective function values at low setting ratios are relatively good, as no large pre- and de-compression angles are desired.

With increasing speeds, the performance at low displacements deteriorates due to cavitation and high-pressure peaks, which can be explained by the throttling effect as presented in sec. 2.2.

\subsection{Optimised Valve Plates with Relative Rotation}

The angular positions of the kidneys for each operating point are visualised in fig. 11 to 13 . The corresponding objective function values are shown in fig. 14 to 15. The most important results are summarised in tab. 4 to 6 .

Both at 1500 and $3000 \mathrm{rpm}$, the results show a great similarity to zero-lapped valve plates. This is motivated by the choice of operating points. Zero-lapped valve plates perform relatively well in operating points with low setting ratios, as commutation takes place at high piston speeds and low pre- and de-compression is desired. As no weighting between the displacement settings has taken place, the influence of the small displacement settings is significant. Furthermore, there is (almost) no relative rotation between the valve plates. Having both valve plates in the same position offers the advantage of being able to use the passage ways on both sides of the pump, which reduces the throttling effect in comparison to using only one passage way (which is the case when relative valve plate rotation takes place). However, the results for the optimised valve plates show reduced flow pulsations and power losses. 


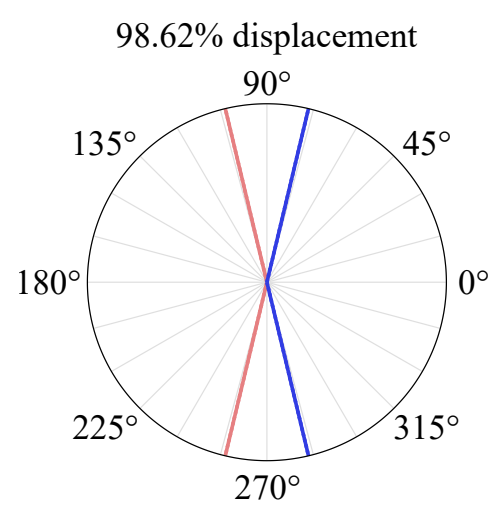

$25 \%$ displacement

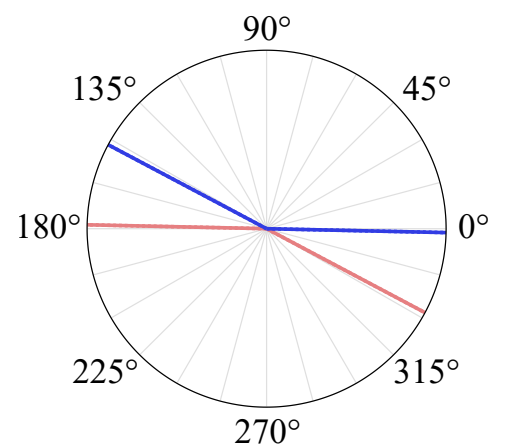

$75 \%$ displacement

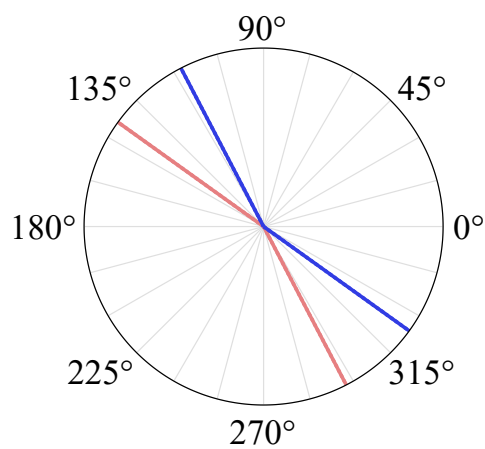

$0 \%$ displacement

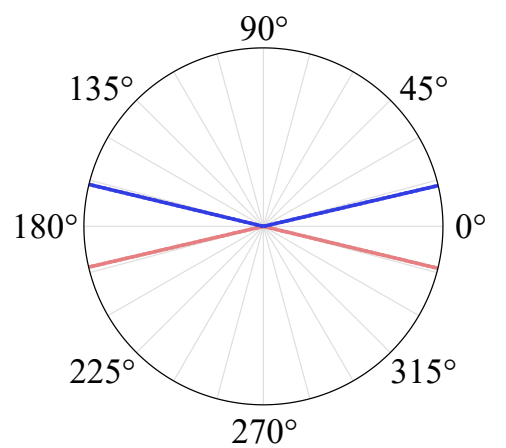

$50 \%$ displacement

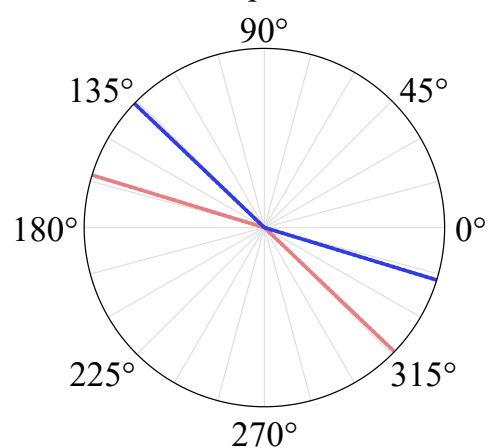

Figure 10: Visualisation of kidney positions of jointly rotated zero-lapped valve plates for each setting ratio at $1500 \mathrm{rpm}$ and $15 \mathrm{MPa}$. The kidney positions differ only marginally between $1500 \mathrm{rpm}$ and $3000 \mathrm{rpm}$, so no additional plots for 3000 rpm are shown. The lines indicate the angular position of the kidney's beginning and end for each setting ratio. The LP kidney is between the blue (dark grey) lines, and the HP kidney is between the red (light grey) lines. BDC is located at $90^{\circ}$, TDC at $270^{\circ}$. The direction of rotation is ccw.

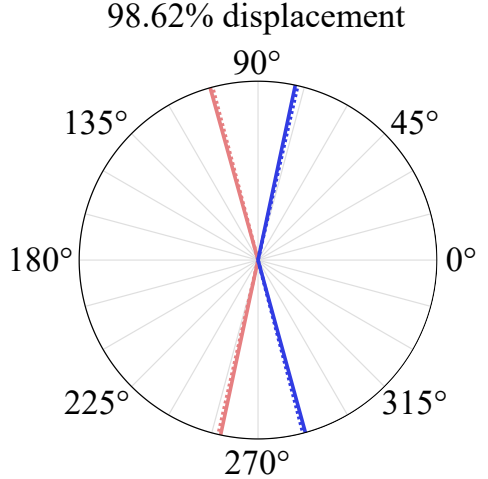

$25 \%$ displacement

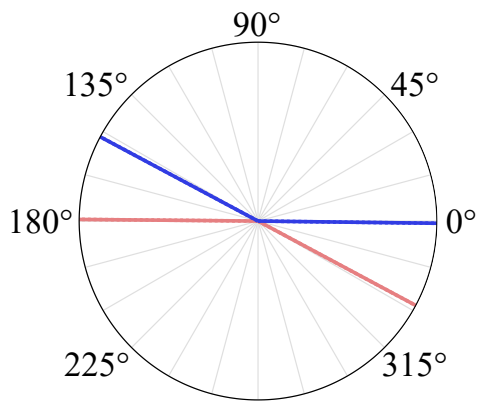

$75 \%$ displacement

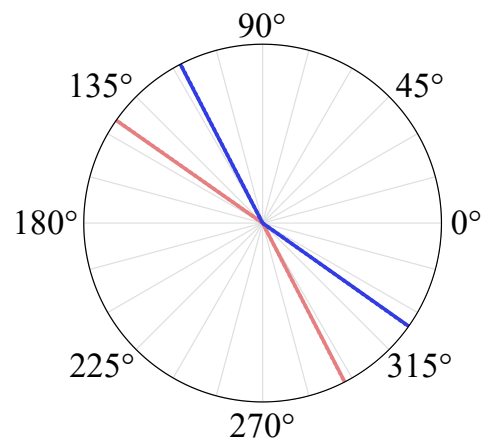

$0 \%$ displacement

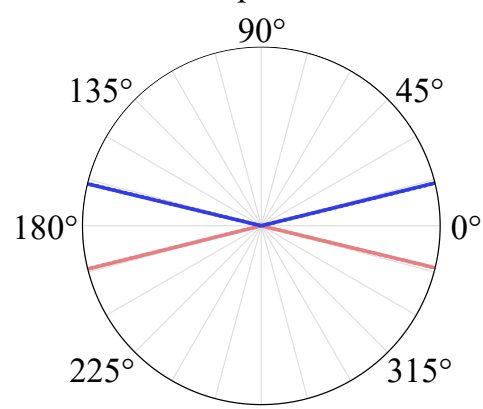

$50 \%$ displacement

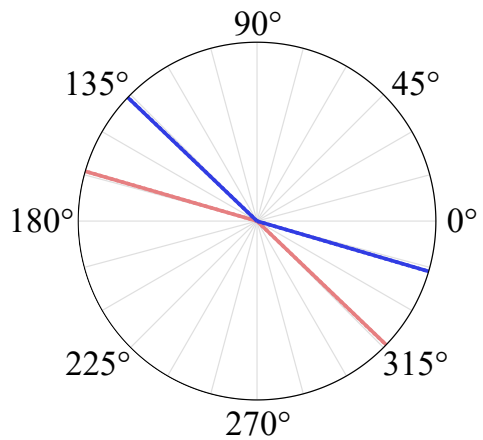

Angular position of ports of valve plate 1 Angular position of ports of valve plate 2 Start/end of HP kidney Start/end of LP kidney

Figure 11: Visualisation of kidney positions which are optimised for valve plate rotation at $1500 \mathrm{rpm}$ and $15 \mathrm{MPa}$, with setting ratios from 0 to $98.62 \%$. The lines indicate the angular position of the kidney's beginning and end for each setting ratio. The LP kidney is between the blue (dark grey) lines, and the HP kidney is between the red (light grey) lines. BDC is located at $90^{\circ}, T D C$ at $270^{\circ}$. The direction of rotation is ccw. As both valve plates are at almost the same position, only one of the can be seen. 

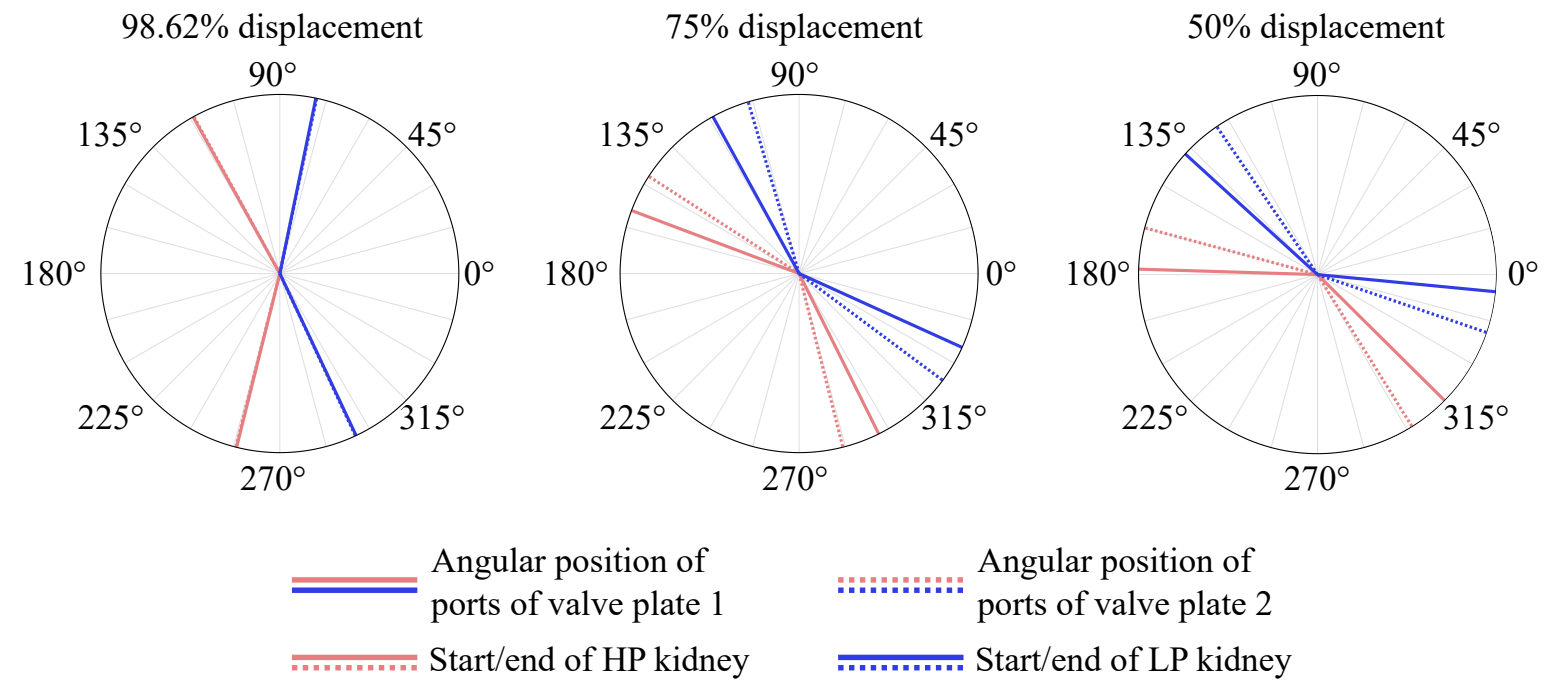

Figure 12: Visualisation of kidney positions which are optimised for valve plate rotation at 1500 rpm and 15 MPa, with setting ratios only from 50 to 98.62\%. The lines indicate the angular position of the kidney's beginning and end for each setting ratio. The LP kidney is between the blue (dark grey) lines, and the HP kidney is between the red (light grey) lines. $B D C$ is located at $90^{\circ}, T D C$ at $270^{\circ}$. The direction of rotation is ccw.

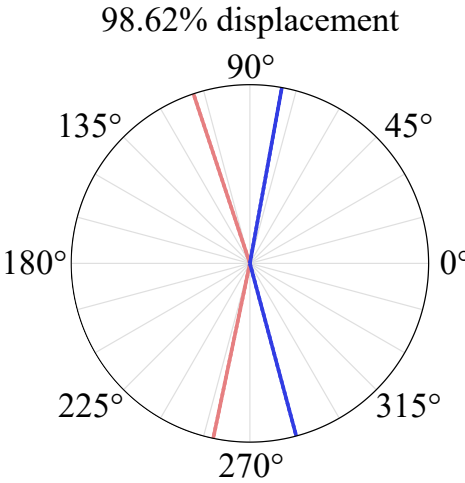

$25 \%$ displacement

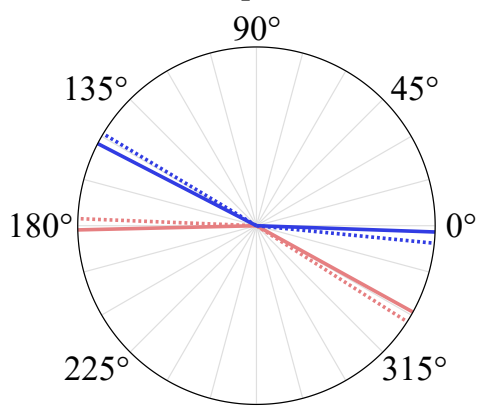

$75 \%$ displacement

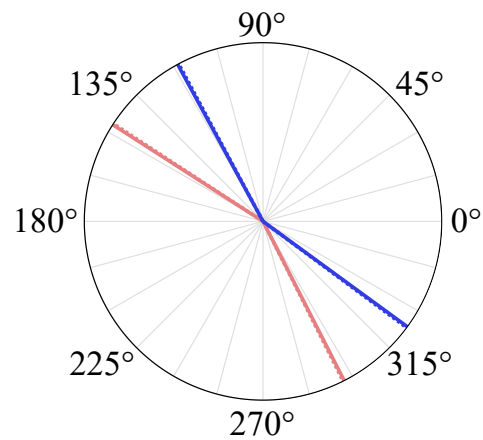

$0 \%$ displacement

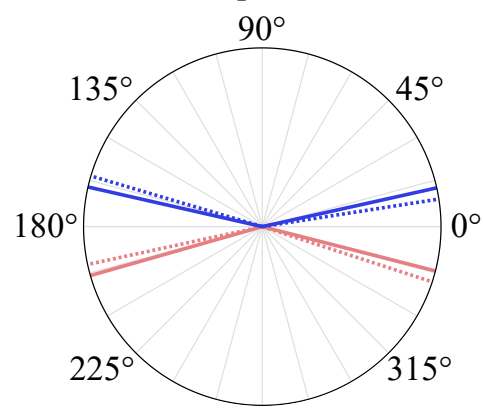

$50 \%$ displacement

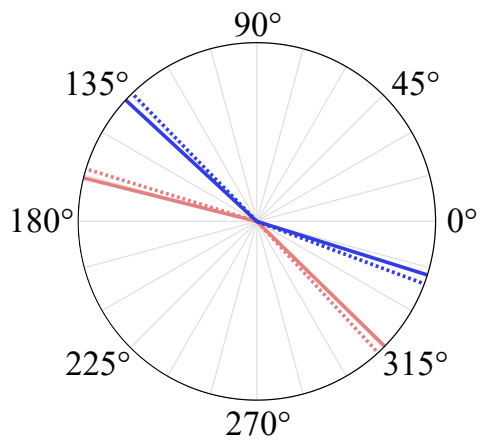

Angular position of ports of valve plate Angular position of ports of valve plate 2 Start/end of HP kidney

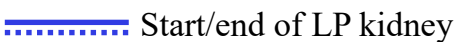

Figure 13: Visualisation of kidney positions which are optimised for valve plate rotation at $3000 \mathrm{rpm}$ and $15 \mathrm{MPa}$. The lines indicate the angular position of the kidney's beginning and end for each setting ratio. The LP kidney is between the blue (dark grey) lines, and the HP kidney is between the red (light grey) lines. BDC is located at $90^{\circ}$, $T D C$ at $270^{\circ}$. The direction of rotation is ccw. 


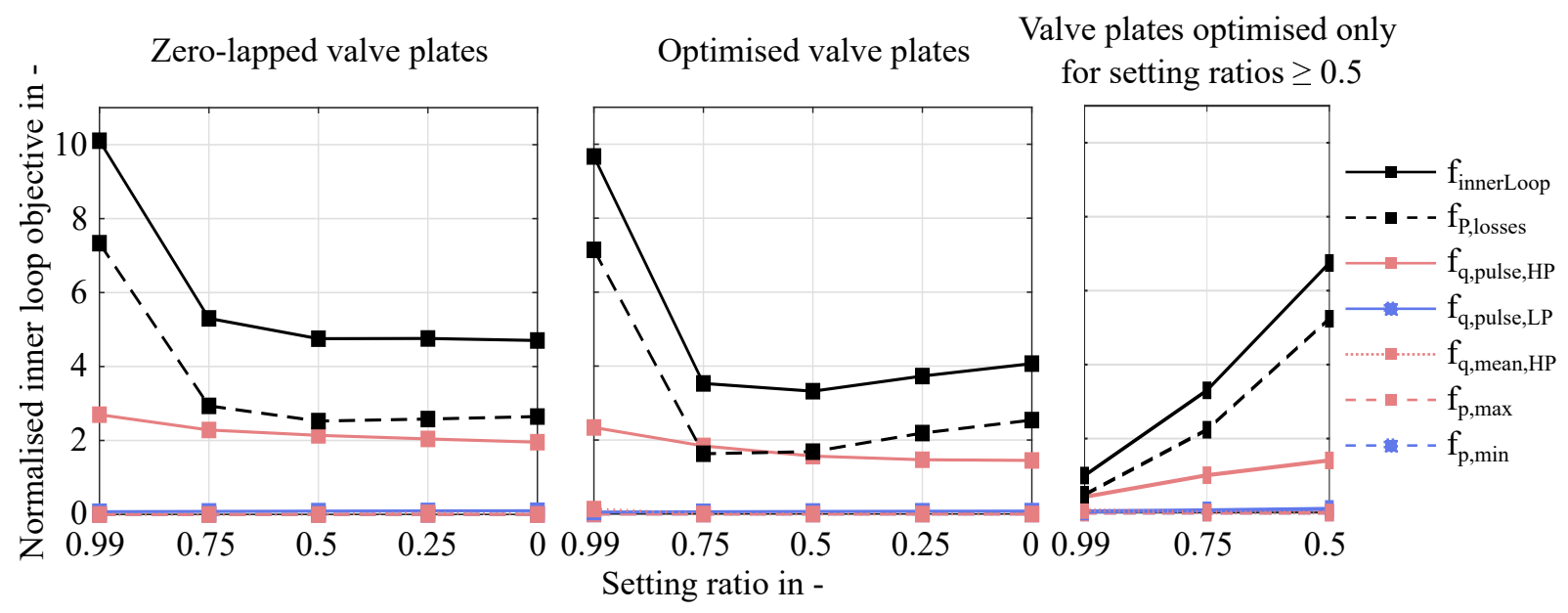

Figure 14: Visualisation of total objective function values and their compositions for each setting ratio for zerolapped valve plates (left), for valve plates optimised for setting ratios from 0.9862 to 0 (center) and for valve plates optimised only for setting ratios from 0.9862 to 0.5 (right) at $1500 \mathrm{rpm}$ and $15 \mathrm{MPa}$. The inner loop objectives are normalised with respect to the lowest inner loop objective for all results presented in this paper, i.e., the lowest normalised objective is 1 and all other objectives are relative to it.

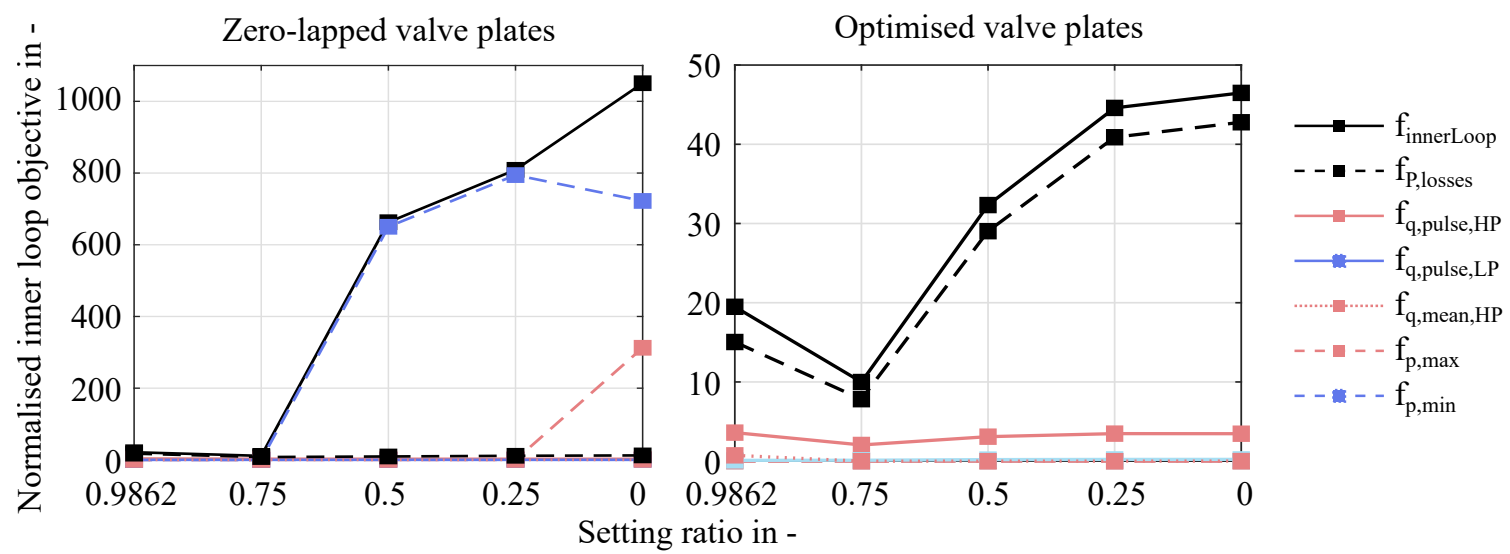

Figure 15: Visualisation of total objective function values and their compositions for each setting ratio for zerolapped valve plates (left) and for optimised valve plates (right) at $3000 \mathrm{rpm}$ and $15 \mathrm{MPa}$. Please mind the individual $y$-axes. The inner loop objectives are normalised with respect to the lowest inner loop objective for all results presented in this paper, i.e., the lowest normalised objective is 1 and all other objectives are relative to it.

The choice and weighting of displacement settings has a significant influence on the optimisation results. For operation at $1500 \mathrm{rpm}$ and $150 \mathrm{bar}$, another optimisation is carried out omitting the lowest setting ratios and only optimising for setting ratios of $0.9862,0.75$ and 0.5 (with equal weights). Naturally, the optimisation results differ from those considering setting ratios down to 0 . The optimisation results and their objective function values are visualised in fig. 12 and 14. The most important numerical values are summarised in tab. 5. The effective bridge angles at full displacement are massively increased, leading to significantly reduced power losses and flow pulsations. For partial displacement, relative valve plate rotation is increased in order to avoid cavitation and highpressure peaks. The effective bridge angles at 50\% displacement are lower than for the solution considering setting ratios down to 0 .

At $3000 \mathrm{rpm}$, objective function values are generally higher than at $1500 \mathrm{rpm}$, as the absolute levels of flow pulsations and power losses are higher. The effective bridge angle used for pre-compression is larger than the effective bridge angle for de-compression. It can also be seen that the more the commutation is moved away from the dead centres, the smaller the effective bridge angles become, so increased relative valve plate rotation occurs. This aligns with the expectations based on sec. 2.1. It is noteworthy that for reduced displacement settings, the effective bridge angle for de-compression allows several degrees of cross-porting, i.e., a direct communication between the HP kidney and LP kidney through the cylinder is enabled. This is necessary, as otherwise cavitation would occur in the chamber, caused by the throttling effect as described in sec. 2.2. Naturally, cross-porting leads to increased losses. The same phenomenon occurs for the effective bridge angle for pre-compression as well, but 
Table 4: Results for zero-lapped and for optimised valve plate in comparison (1500 rpm, $15 \mathrm{MPa}$ ).

\begin{tabular}{|c|c|c|c|c|c|c|c|c|c|c|}
\hline \multirow[b]{2}{*}{ Setting ratio in - } & \multicolumn{5}{|c|}{ Zerolapped valve plate } & \multicolumn{5}{|c|}{ Optimised valve plate } \\
\hline & 0.9862 & 0.75 & 0.5 & 0.25 & 0 & 0.9862 & 0.75 & 0.5 & 0.25 & 0 \\
\hline$\varphi_{\text {ref }}$ in deg & & & 76.6 & & & & & 77.2 & & \\
\hline$\Psi_{1}$ in deg & $\ldots$ & $\ldots \varphi_{c}$ & $m b e r=26$ & & & & & 27.6 & & \\
\hline$\Psi_{2}$ in deg & & & 180 . & & & & & 179.9 & & \\
\hline$\Psi_{3}$ in deg & $\ldots$. & $\ldots \varphi_{c}$ & $m b e r=26$ & & & & & 27.4 . & & \\
\hline$\Psi_{4}$ in deg & 0 & 40.8 & 59.7 & 75.3 & 89.8 & 0 & 40.2 & 59.1 & 74.7 & 89.2 \\
\hline$\Psi_{5}$ in deg & & 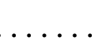 & $0 \ldots$ & & & 0.8 & 0.1 & 0.0 & 0.0 & 0.1 \\
\hline$\varphi_{\mathrm{b}, \mathrm{pc}}$ in deg & & $\ldots \varphi_{c}$ & $m b e r=26$ & & & 26.8 & 27.5 & 27.5 & 27.5 & 27.5 \\
\hline$\varphi_{\mathrm{b}, \mathrm{dc}}$ in deg & $\ldots \ldots$ & $\ldots \varphi_{c}$ & $m b e r=26$ & & & 26.6 & 27.4 & 27.4 & 27.4 & 27.4 \\
\hline$p_{\max }$ in $\mathrm{MPa}$ & 15.01 & 15.34 & 15.72 & 16.07 & 16.32 & 15.01 & 15.43 & 16.12 & 17.04 & 17.99 \\
\hline$p_{\min }$ in $\mathrm{MPa}$ & 0.490 & 0.283 & 0.188 & 0.159 & 0.175 & 0.490 & 0.234 & 0.096 & 0.060 & 0.093 \\
\hline$q_{\text {mean,HP }}$ in $1 / \mathrm{min}$ & 51.78 & 39.38 & 26.25 & 13.13 & 0.00 & 51.76 & 39.38 & 26.25 & 13.13 & 0.00 \\
\hline$q_{\text {pulse,HP }}$ in $1 / \mathrm{min}$ & 36.9 & 31.2 & 29.3 & 28.0 & 26.7 & 32.0 & 25.2 & 21.4 & 20.1 & 19.8 \\
\hline$q_{\text {pulse,LP }}$ in $1 / \mathrm{min}$ & 19.2 & 21.3 & 23.3 & 24.9 & 26.0 & 13.7 & 15.7 & 17.8 & 19.4 & 20.3 \\
\hline$P_{\text {loss }}$ in $\mathrm{W}$ & 190.8 & 76.3 & 65.7 & 67.1 & 68.8 & 185.8 & 42.4 & 43.9 & 56.9 & 66.0 \\
\hline$f_{\text {normalised }}$ in - & 10.1 & 5.3 & 4.8 & 4.8 & 4.7 & 9.7 & 3.5 & 3.3 & 3.7 & 4.1 \\
\hline
\end{tabular}

Table 5: Results for valve plates optimised only for setting ratios from 0.9862 to 0.5 at 1500 rpm and 15 MPa.

\begin{tabular}{|c|c|c|c|}
\hline \multirow[b]{2}{*}{ Setting ratio in - } & \multicolumn{3}{|c|}{ Optimised valve plate } \\
\hline & 0.9862 & 0.75 & 0.5 \\
\hline$\varphi_{\text {ref }}$ in deg & $\ldots$ & 78.1 & \\
\hline$\Psi_{1}$ in deg & $\ldots$ & 40.6 & \\
\hline$\Psi_{2}$ in deg & $\ldots \ldots$ & 177.6 & \\
\hline$\Psi_{3}$ in deg & $\ldots$ & 39.2 & \\
\hline$\Psi_{4}$ in deg & 0 & 28.4 & 46.1 \\
\hline$\Psi_{5}$ in deg & 0.3 & 12.3 & 13.4 \\
\hline$\varphi_{\mathrm{b}, \mathrm{pc}}$ in deg & 40.4 & 28.3 & 27.2 \\
\hline$\varphi_{\mathrm{b}, \mathrm{dc}}$ in $\mathrm{deg}$ & 39.0 & 26.9 & 25.8 \\
\hline$p_{\max }$ in $\mathrm{MPa}$ & 15.29 & 17.07 & 17.33 \\
\hline$p_{\min }$ in $\mathrm{MPa}$ & 0.050 & 0.084 & 0.050 \\
\hline$q_{\text {mean,HP }}$ in $1 / \mathrm{min}$ & 51.77 & 39.38 & 26.25 \\
\hline$q_{\text {pulse,HP in } 1 / \mathrm{min}}$ & 5.8 & 13.8 & 19.3 \\
\hline$q_{\text {pulse,LP }}$ in $1 / \mathrm{min}$ & 7.9 & 15.1 & 25.5 \\
\hline$P_{\text {loss }}$ in $\mathrm{W}$ & 12.6 & 58.2 & 136.3 \\
\hline$f_{\text {normalised }}$ in - & 1 & 3.3 & 6.7 \\
\hline
\end{tabular}

less profound.

With decreasing displacement, the losses increase massively. Therefore, the objective function value of the losses dominates the optimisation for these setting ratios (see fig. 15 and tab. 6).

\section{Discussion}

For $1500 \mathrm{rpm}$, the optimisation results in a valve plate design which is similar to a zero-lapped valve plate, but offers slightly improved performance over the whole operating range. This is motivated by the choice and weighting of operating points. At low setting ratios, a zero-lapped valve plate performs quite well, because cross-porting is avoided, and no pre- and de-compression takes place (which could lead to cavitation and high-pressure peaks). This deteriorates the performance at high setting ratios, as pre-compression cannot be provided.

When omitting the lower setting ratios, performance at full displacement is drastically improved with decreased power losses and flow pulsations, see fig. 14 and tab. 4 and 5. This is enabled by a massive increase of the effective bridge angles. However, the performance at the $75 \%$ and $50 \%$ setting ratios slightly declines. This is caused by a more profound throttling effect due to larger relative valve plate rotation. Relative valve plate rotation reduces the passage area for the fluid around commutation, which hinders the flow into/out of the chamber. Therefore, 
Table 6: Results for zero-lapped and for optimised valve plate in comparison (3000 rpm, $15 \mathrm{MPa}$ ).

\begin{tabular}{|c|c|c|c|c|c|c|c|c|c|c|}
\hline \multirow[b]{2}{*}{ Setting ratio in - } & \multicolumn{5}{|c|}{ Zerolapped valve plate } & \multicolumn{5}{|c|}{ Optimised valve plate } \\
\hline & 0.9862 & 0.75 & 0.5 & 0.25 & 0 & 0.9862 & 0.75 & 0.5 & 0.25 & 0 \\
\hline$\varphi_{\text {ref }}$ in deg & & & 76.6 & & & & & 79.7 & & \\
\hline$\Psi_{1}$ in deg & & $\ldots \varphi_{c l}$ & $m b e r=26$ & & & & & 28.7 & & \\
\hline$\Psi_{2}$ in deg & & $\ldots \ldots$ & 180 . & & & & & 178.5 & & \\
\hline$\Psi_{3}$ in deg & $\ldots \ldots$ & $\ldots \varphi_{c l}$ & mber $=2($ & & & & & 26.9 . & & \\
\hline$\Psi_{4}$ in deg & 0 & 41.0 & 60.0 & 75.6 & 90.1 & 0.0 & 38.4 & 54.5 & 69.3 & 83.8 \\
\hline$\Psi_{5}$ in deg & & $\cdots$ & $0 \ldots$ & & & 0.0 & 0.4 & 3.0 & 3.6 & 3.7 \\
\hline$\varphi_{\mathrm{b}, \mathrm{pc}}$ in deg & & $\ldots \varphi_{c l}$ & mber $=26$ & & & 28.7 & 28.3 & 25.7 & 25.1 & 25.0 \\
\hline$\varphi_{\mathrm{b}, \mathrm{dc}} \mathrm{i}$ & & $\ldots \varphi_{c l}$ & $m b e r=26$ & & & 26.9 & 26.5 & 23.9 & 23.3 & 23.2 \\
\hline$p_{\max }$ in $\mathrm{MPa}$ & 15.04 & 15.80 & 16.79 & 17.72 & 18.41 & 15.04 & 18.00 & 17.69 & 17.75 & 18.00 \\
\hline $\mathrm{MPa}$ & 0.459 & 0.079 & 0.005 & 0.001 & 0.003 & 0.459 & 0.088 & 0.050 & 0.050 & 0.050 \\
\hline$q_{\text {mean,HP }}$ in $1 / \mathrm{min}$ & 103.55 & 78.75 & 52.50 & 26.25 & 0.00 & 103.49 & 78.75 & 52.50 & 26.25 & 0.00 \\
\hline in $1 / \min$ & 52.9 & 43.1 & 41.9 & 41.7 & 41.1 & 49.3 & 28.0 & 42.3 & 47.6 & 47.5 \\
\hline & 27.4 & 31.3 & 33.4 & 35.6 & 37.4 & 27.2 & 31.1 & 51.5 & 57.8 & 59.3 \\
\hline$P_{\text {loss }} \mathrm{i}$ & 452.2 & 207.0 & 247.5 & 297.8 & 325.4 & 391.2 & 203.5 & 755.0 & 1063 & 1113 \\
\hline$f_{\text {normalised }}$ in - & 21.4 & 11.3 & 663 & 809 & 1051 & 19.5 & 10.0 & 32.3 & 44.6 & 42.8 \\
\hline
\end{tabular}

minimal pressures decrease, maximum pressures increase and higher losses occur. Due to the more profound throttling effect, cross-porting even needs to be applied at a $50 \%$ setting ratio in order to maintain the required minimum pressure level.

The results at $1500 \mathrm{rpm}$ indicate that valve plate rotation is not capable of providing an efficient and silent pump from zero to full displacement. However, it can be used to improve the performance for a specific range of setting ratios.

For $3000 \mathrm{rpm}$, the optimisation also results in a valve plate design which is similar to a zero-lapped valve plate, but offers slightly improved performance at high setting ratios compared to zero-lapped valve plates. At low setting ratios, the zero-lapped valve plates suffer from cavitation and high pressure peaks. Due to relative valve plate rotation, the optimised solution can avoid cavitation and high-pressure peaks. However, several degrees of crossporting are applied in order to maintain the required pressure levels in the chamber. This leads to very high power losses. Increased boost pressures at the pump inlet will only partially reduce the issues caused by the throttling effect. Furthermore, reducing the throttling effect by providing larger passage areas for the fluid is challenging, as the fluid flows through the piston, slipper and valve plate, see fig. $1 \mathrm{a}$.

The pre-compression angles are chosen to be larger than the de-compression angles, which is in alignment with the expectations based on sec. 2.1 .

Both at $1500 \mathrm{rpm}$ and $3000 \mathrm{rpm}$, the mean flow level at full displacement is slightly reduced in comparison to the zero-lapped valve plates, so the soft constraint is slightly violated. This indicates that a minor reduction of the flow demand at full displacement can offer more freedom to design valve plates for reduced losses and pulsations.

\section{Conclusion}

Valve plate rotation can control the displacement of an axial piston machine. However, cavitation and high pressure peaks in the chamber are a drawback at low setting ratios and high speeds. This drawback can be overcome by using a double pump with opposing pistons, which has two valve plates. A relative rotation between the two valve plates leads to variable bridge angles. These variable bridge angles can eliminate the risk of cavitation and high pressure peaks, at the expense of increased power losses (and cross-porting).

Valve plate rotation seems mainly suitable for machines which mostly run at high setting ratios but need the ability to survive low setting ratios.

\section{Acknowledgement}

This research was funded by the Swedish Energy Agency (Energimyndigheten, Grant Number 50181-1). 


\section{Nomenclature}

\begin{tabular}{ccc}
\hline Designation & Denotation & Unit \\
\hline$f$ & Objective function value & - \\
$g$ & Linear weighting factor & varies between equations \\
$h$ & Weighting factor in exponent & varies between equations \\
$p$ & Pressure & $\mathrm{Pa}$ \\
$p_{0}$ & Reference pressure & $\mathrm{Pa}$ \\
$q$ & Flow & $\mathrm{m}^{3} / \mathrm{s}$ \\
$t$ & Time & $\mathrm{s}$ \\
$x_{0}$ & Fraction of undissolved air at reference pressure $p_{0}$ & - \\
$A_{\mathrm{p}}$ & Piston area & $\mathrm{m}$ \\
$P_{\text {loss }}$ & Power losses & $\mathrm{W}$ \\
$R_{\mathrm{b}}$ & Barrel radius & $\mathrm{m}$ \\
$V_{\mathrm{cyl}}$ & Dead volume & $\mathrm{m}$ \\
$V_{\mathrm{dead}}$ & Swash plate angle & $\mathrm{m}$ \\
$\alpha$ & Effective bulk modulus & $\mathrm{deg}$ \\
$\beta_{\mathrm{e}}$ & Oil's bulk modulus & $\mathrm{Pa}$ \\
$\beta_{\mathrm{oil}}$ & Machine setting ratio & $\mathrm{Pa}$ \\
$\varepsilon$ & Shaft/barrel angle & - \\
$\varphi$ & Displacement volume of one chamber & $\mathrm{rad}$ \\
$\varphi_{\text {chamber }}$ & Swept shaft angle covered by one displacement chamber & $\mathrm{deg}$ \\
$\varphi_{\mathrm{b}, \mathrm{dc}}$ & Effective bridge angle at de-compression & $\mathrm{deg}$ \\
$\varphi_{\mathrm{b}, \mathrm{pc}}$ & Effective bridge angle at pre-compression & $\mathrm{deg}$ \\
$\varphi_{\mathrm{ref}}$ & Reference angle for kidney angles & $\mathrm{deg}$ \\
$\varphi_{\mathrm{VP}}$ & Valve plate rotation angle & $\mathrm{deg}$ \\
$\kappa$ & Isentropic expansion factor of air & - \\
$\omega$ & Shaft/barrel angular speed & $\mathrm{rad} / \mathrm{s}$ \\
$\Psi_{1}$ & Bridge angle between closing LP kidney and opening HP kidney & $\mathrm{deg}$ \\
$\Psi_{2}$ & Angle between closing of LP kidney and closing of HP kidney & $\mathrm{deg}$ \\
$\Psi_{3}$ & Bridge angle between closing HP kidney and opening LP kidney & $\mathrm{deg}$ \\
$\Psi_{4}$ & Joint rotation angle of both valve plates & $\mathrm{deg}$ \\
$\Psi_{5}$ & Relative rotation angle between valve plate 1 and valve plate 2 & $\mathrm{deg}$ \\
\hline & & \\
\hline
\end{tabular}

\begin{tabular}{cc}
\hline Abbreviation & Meaning \\
\hline $\mathrm{ccw}$ & counter-clockwise \\
$\mathrm{cw}$ & clockwise \\
BDC & Bottom Dead Center \\
HP & High Pressure \\
LP & Low Pressure \\
TDC & Top Dead Center \\
\hline
\end{tabular}




\section{References}

[1] Jaroslav Ivantysyn and Monika Ivantysynova. Hydrostatic pumps and motors : principles, design, performance, modelling, analysis, control, and testing. New Delhi: Akademia Books International, 2001. ISBN: 8185522162.

[2] Liselott Ericson, Samuel Kärnell and Martin Hochwallner. "Experimental Investigation of a Displacementcontrolled Hydrostatic Pump/Motor by Means of Rotating Valve Plate". In: Proceedings of 15:th Scandinavian International Conference on Fluid Power, (SICFP'17), Linköping, Sweden. Linköping University Electronic Press, 2017. DOI: 10.3384/ecp1714419.

[3] P.A.J. Achten, Z. Fu and G.E.M. Vael. "Transforming future hydraulics : a new design of a hydraulic transformer". In: The Fifth Scandinavian International Conference on Fluid Power (SICFP '97), Sweden, Linköping. 1997.

[4] Peter A. J. Achten and Zhao Fu. "Valving Land Phenomena of the Innas Hydraulic Transformer". In: International Journal of Fluid Power 1.1 (2000), pp. 39-47. DOI: 10.1080/14399776 . 2000 . 10781081.

[5] Junhee Cho, Xiaoping Zhang, Noah D. Manring and Satish S. Nair. "Dynamic Modelling and Parametric Studies of an Indexing Valve Plate Pump". In: International Journal of Fluid Power 3.3 (2002), pp. 37-48. DOI: $10.1080 / 14399776.2002 .10781146$.

[6] Andreas Tonnqvist, Jonas Forssell, Jan-Ove Palmberg, Liselott Ericson and Anders Hedebjörn. "Variable Pre and De-Compression Control Mechanism and Method for Hydraulic Displacement Pump". Pat. US10968741B2. 2021.

[7] Noah D. Manring. Fluid Power Pumps and Motors: Analysis, Design and Control. McGraw Hill Book CO, 2013. ISBN: 0071812202 .

[8] Herbert E. Merritt. Hydraulic Control Systems. New York: John Wiley and Sons, Inc., 1967. ISBN: 0471596175.

[9] Maria Pettersson. Design of fluid power piston pumps : with special reference to noise reduction. Dissertation. Linköping: Division of Fluid Power Technology, Department of Mechanical Engineering, Linköping University, 1995. ISBN: 917871592x.

[10] M. J. Box. "A New Method of Constrained Optimization and a Comparison With Other Methods". In: The Computer Journal 8.1 (1965), pp. 42-52. DOI: 10.1093/comjnl/8.1.42.

[11] Petter Krus and Johan Andersson. “Optimizing Optimization for Design Optimization”. In: Volume 2: 29th Design Automation Conference, Parts A and B. ASMEDC, 2003. DOI: 10.1115/detc2003/dac-48803.

[12] Liselott Ericson, Johan Ölvander and Jan-Ove Palmberg. "On optimal design of hydrostatic machines". In: Proceedings of the 6th International Fluid Power Conference, IFK, Vol WS. 2008, pp. 273-286. 